\title{
A case report on MDS - MPD overlap syndrome - a diagnostic dilemma
}

\author{
Omhare $\mathrm{A}^{1}$, Singh $\mathrm{SK}^{2}$, Srivastava $\mathrm{K}^{3}$, Mishra $\mathrm{V}^{4}$, Vahikar $\mathrm{SU}^{5}$ \\ ${ }^{1}$ Dr Anita Omhare, Pathology, Lecturer, GSVM Medical College, Kanpur, Former SR St. Stephen's College, New Delhi, \\ ${ }^{2}$ Dr Sanjeev Kumar Singh, Pathology, Assistant Professor. UPRIMS, Etawah, ${ }^{3}$ Dr Kanchan Srivastava, Pathology, \\ Assistant Professor, BRD Medical College, Gorakhpur, Former Lecturer, GSVM Medical College, Kanpur, ${ }^{4}$ Dr Vandana \\ Mishra, MD (Pathology), Lecturer, GSVM Medical College, Kanpur, ${ }^{5}$ Dr Shilpa U. Vahikar, Pathology, Associate \\ Professor, BRD Medical College, Gorakhpur
}

Address for Correspondence: Dr Kanchan Srivastava, Assistant Professor, Pathology, BRD Medical College, Gorakhpur. E-mail: kanchanshrivastava11@gmail.com

\begin{abstract}
Refractory anemia with ring sideroblasts and thrombocytosis (RARS - T) is a rare disease with a controversial status which presents a diagnostic dilemma as it shows overlapping clinical, laboratory and morphological features of two distinct entities-myelodysplastic syndrome (MDS) and myeloproliferative neoplasms (MPN). In WHO classification RARS-T is included in MDS/MPN-U category. We report a case of 54 years female who presented with weakness, intermittent fever, anemia along with thrombocytosis. Hematological workup revealed hypercellular marrow with abnormal megakaryocytes and presence of ring sideroblasts (46\%). Further, presence of JAK2 V617F mutation indicated an underlying MPN and presence of ring sideroblasts an element of MDS. RARS-T was first defined in 2001 as an overlap syndrome. Whether RARS-T is a separate clinical entity or a result of additional acquired genetic defect resulting in progression of RARS/ Essential Thrombocytosis (ET) is still a topic for debate. Hence this unusual case emphasizes need for awareness of this entity among clinicians and pathologists to enable its accurate diagnosis and appropriate management.
\end{abstract}

Key words: Myelodysplastic, Myeloproliferative, Thrombocytosis, Sideroblasts

\section{Introduction}

The classification of hematologic malignancies has evolved over the years from acute myeloid and lymphoid leukemias in the $\mathrm{FAB}$ classification based on morphology alone to the present day WHO classification based on cell morphology, immunophenotyping and cytogenetics. Three main categories of myeloid neoplasms are the acute leukemias, the chronic myeloproliferative disorders and the myelodysplastic syndrome $\{$ WHO classification [1]\}. It is now well recognized that there are some disorders which show one or more features of these three categories. There are some overlap syndrome disorders which show one or more features of these three categories which sometimes presents a diagnostic dilemma and WHO classified these disorders as

Manuscript received: $30^{\text {th }}$ January 2017

Reviewed: $6^{\text {th }}$ February 2017

Author Corrected: $14^{\text {th }}$ February 2017

Accepted for Publication: $21^{\text {st }}$ February 2017
Myelodysplastic /Myeloproliferative Neoplasm [1]. Treatment modalities for these overlap syndromes are also not well defined. RARS-T is included in the category of MDS/MPN Neoplasm Unclassifiable, which is characterized by some signs of essential thrombocytosis including marked increase in platelet count, hypercellular marrow and increased megakaryocytes, but also have ring sideroblasts, a feature associated with MDS [1]. The criteria for diagnosis were platelet count more than $450 \times 10^{9} / \mathrm{L}$, erythroid dysplasia, $>15 \%$ ringed sideroblasts, $<5 \%$ undifferentiated marrow blasts and megakaryocytic proliferation with morphology pattern similar to essential thrombocytosis, with exclusion of chromosomal abnormalities 5q- syndrome or rearrangement of the long arm of chromosome 3 [2]. Whether this is a distinct entity of RARS spectrum or the two separate disorders in the same patient is not yet 
clear, so that the designation of MDS/MPD-U is appropriate until future studies indicate a more exact classification [1]. We report a case of a middle aged woman with MDS/MPD overlap syndrome with coexistence of features of both these entities with positive JAK2V617F mutation.

\section{Case Report}

A 54 year old female with a history of hypertension, hypothyroidism and hyperuricemia, on and off low grade fever, weakness and loss of appetite for past one year was admitted in our hospital with complaints of lower backache with radiating pain down to both lower limbs for one month. Physical examination revealed tenderness over the lower spinal vertebrae at L4-L5 level and splenomegaly ( $3 \mathrm{~cm}$ below the left costal margin) and no hepatomegaly. Her complete blood count revealed total leukocytes count $12.4 \times 10 \%$ L, Differential count showed $88 \%$ polymorphs, $9 \%$ lymphocytes, $2 \%$ eosinophils, $1 \%$ monocytes. Hemoglobin-10.5 gm/dl, MCV-77.5fl, MCH-24.2pg, MCHC-31.2g/dl, RDW-33.5\% and platelet count $-523 \times 10^{9} / \mathrm{L}$. The Leishman stained peripheral blood smear showed double population of RBCs, one microcytic, hypochromic and the other normocytic, normochromic with moderate anisocytosis and basophilic stippling.

Neutrophils showed dysplasia in the form of hypogranularity and abnormal chromatin. There was thrombocytosis with platelet anisocytosis. No blasts were seen. (Figure 1) Serum B12, folic acid, iron, ferritin, ESR, liver and kidney test were in normal range except serum uric acid which was slightly raised $-8.5 \mathrm{mg} / \mathrm{dl}$. CRP and other acute phase reactants were evaluated and results were within normal range. Bone marrow aspiration and biopsy were performed to know the cause of splenomegaly. Bone marrow smears revealed hypercellularity with moderate degree of erythroid hyperplasia with normoblastic maturation except few late normoblasts which showed mild dysplastic features. Clusters of erythroid precursors comprised approximately $37.5 \%$ of total nucleated bone marrow cells. Myeloid to erythroid ratio was 1.6:1.

The myeloid series showed all stages of maturation with left shift and increase in myeloblasts (05\%) and mild dysplastic features in neutrophils. Lymphocytes were normal. The most prominent feature was megakaryocytes with marked proliferation and hyperlobulated nuclei arranged in loose clusters. Occasional clusters of osteoblasts were seen (Figure 3) Prussian blue iron stain disclosed moderate increase in iron stores in bone marrow fragments and $46 \%$ ringed sideroblasts. (Figure 4) Bone marrow biopsy specimen revealed hypercellular marrow spaces, erythroid hyperplasia and increased number of megakaryocytes with hyperlobulated nuclei arranged in loose clusters. (Figure 5) Reticulin stain showed increased reticulin fibers.(Figure 6) LAP score was normal. Based on these finding a probable diagnosis of MDS/MPD Overlap syndrome was made, which was further confirmed by radiological findings and special investigations done at higher referral centre. MRI T2 weighted images taken through the lumbar vertebrae showed the features of myeloproliferative disorder that is the replacement of fatty components of vertebral marrow resulted in dark signal intensity from the vertebral body. Axial images taken through L4-L5 vertebral disc showed a small annular tear at the periphery of the disc which could be the probable cause for lower backache. (Figure 2) Investigations done at higher referral centre showed negative BCR-ABL p210 $\mathrm{t}(9 ; 22)$ (q34;q11) translocation, marker of chronic myeloid leukemia (CML) but positive JAK2V617F mutation typically present in polycythemia vera, as well as in $50 \%$ cases of essential thrombocytosis and primary myelofibrosis (PMF). Cytogenetic analysis for the detection of deletion of the long arm of the 5th chromosome was not done. However the morphology of megakaryocytes and presence of marrow blasts in our case were not consistent with 5q- syndrome. So a final diagnosis of RARS-T was made.

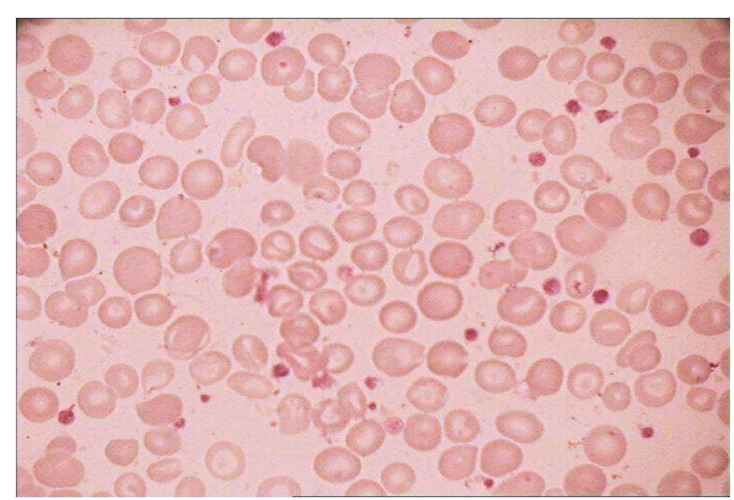

Figure-1

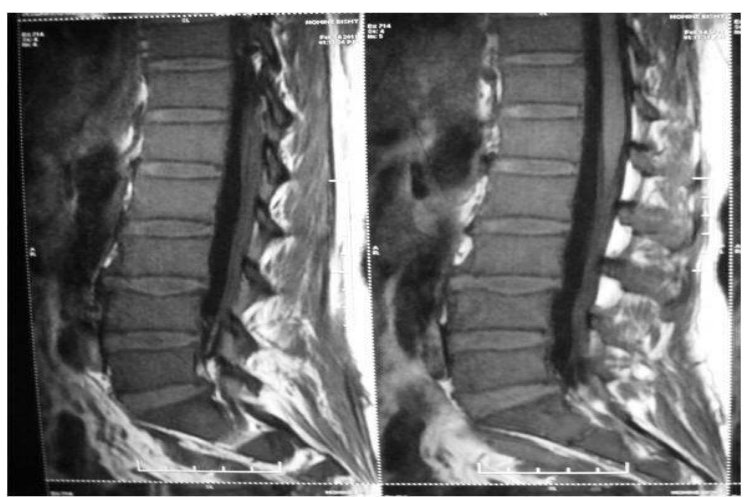

Figure-2 


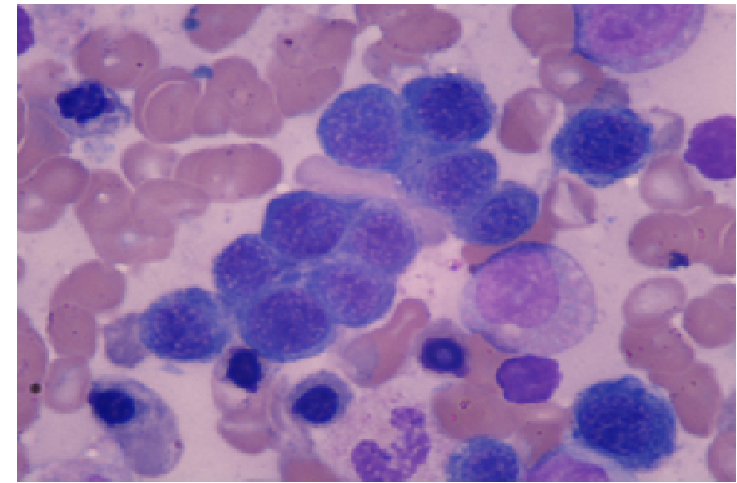

Figure-3

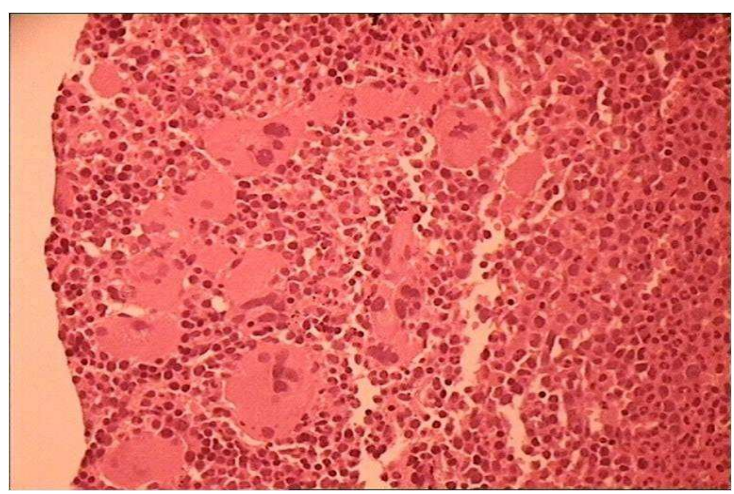

Figure-5

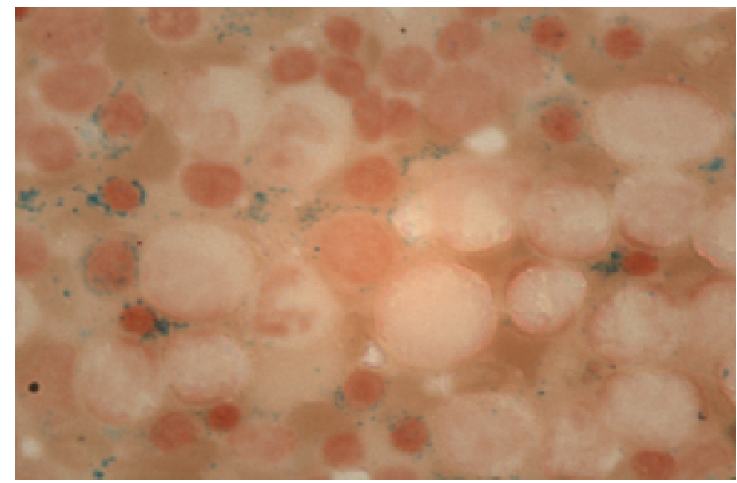

Figure-4

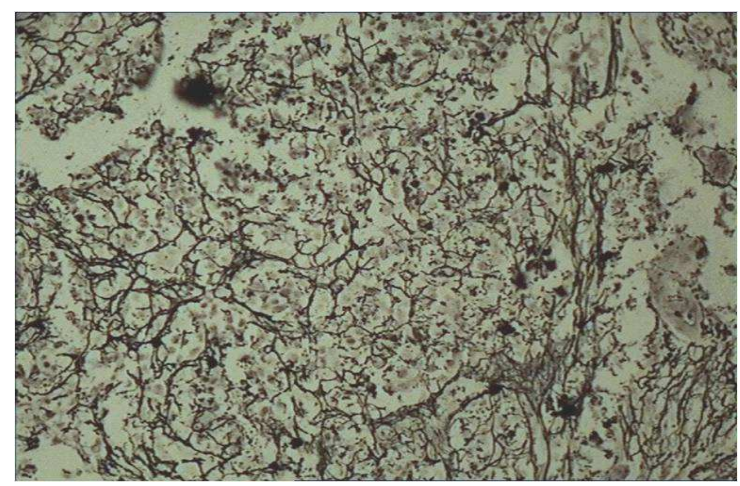

Figure-6

\section{Legends to Figure}

Figure 1 - Photomicrograph (Leishman stain, 400X) showing RBC anisocytosis and thrombocytosis

Figure 2 - MRI through the Lumbar region

Figure 3 - Microphotograph (H and E, 400X) of bone marrow smears showing mild dyserthropoisis.

Figure 4 _ Microphotograph (Prussian blue iron stain, 400X) showing ring sideroblasts

Figure 5 - Microphotograh (H and E, 100X) showing hypercellular marrow with increased abnormal megakaryocytes Figure 6- Microphotograph (Reticulin stain 400X) showing increased Reticulin fibres

\section{Discussion}

This case showed overlapping features of both myelodysplastic syndrome and myeloproliferative disorders. MDS is characterized by peripheral cytopenia and hypercellular bone marrow with ineffective haematopoiesis whereas MPDs are characterized by proliferation of one or more myeloid (granulocytic, erythroid or megakaryocytic) lineages [1]. So in this case we ruled out other conditions which have almost similar findings including primary myelofibrosis, polycythemia vera, CML, MDS with isolated deletion $5 \mathrm{q}$, essential thrombocytosis and their combinations. Thrombocytosis associated with hypercellular bone marrow along with marked proliferation of megakaryocytes and mildly increased fibrosis is suggestive of early stage of primary myelofibrosis but presence of ringed sideroblasts, dysplastic features in granulocytic and erythroid series and positive JAK2
V617F mutation as shown in present case are against the diagnosis of PMF [1,5]. Presence of low haemoglobin, low hematocrit and low red cell mass in present case excludes the possibility of Polycythemia vera [4]. There was no clinical, morphological and cytogenetic features (absent LAP score, negative BCR$\mathrm{ABL}$ ) favoring CML.

Another condition associated with clonal thrombocytosis is MDS with $5 \mathrm{q}$ deletion syndrome characterized by presence of increased number of small or normal sized megakaryocytes with monolobated or hypolobated nuclei, usually placed eccentrically and percentage of blasts in blood and bone marrow should be less than $5 \%$ [1]. In present case cytogenetic analysis for $5 q$ deletion was not done. However the morphology of megakaryocytes was against MDS with $5 \mathrm{q}$ deletion 
[1]. The presence of marked proliferation of megakaryocytes with hyperlobulated nuclei arranged in loose clusters was in favor of Essential thrombocytosis but dysplasia in erythroid and granulocytic series and presence of ringed sideroblasts indicated presence of some other component of MDS. So a provisional diagnosis of MDS/MPN-U was kept which was then further confirmed by additional investigations. In 2005, Shaw GR coined the term "ringed sideroblasts with thrombocytosis" (RST) and he included patients with platelet count more than $500 \times 10^{9} / \mathrm{L}$ and normal conventional cytogenetics study, with no evidence of iron deficiency or splenectomy or other causes of reactive thrombocytosis and other secondary causes for ringed sideroblasts with more than $3 \%$ blood and marrow blasts [5]. But now it has been reported by many working groups that there is strong association of RARS-T with high frequency of JAK2V617F mutation [6]. In contrast to all other MPD/MDS cases where JAK 2 mutation is positive in a minorities of cases, upto $60 \%$ RARS-T cases are JAK2 positive [5]. JAK2 mutation positive patient have more favorable prognosis than patients without the JAK2 mutation and JAK2 mutation positive cases had significantly higher red blood cell count and hemoglobin level, lower mean corpuscular volume and higher leukocyte count [7]. So in the present case, findings of an increased percentage of ringed sideroblasts, presence of myeloblasts in bone marrow, megakaryocytic hyperplasia, mild anemia, thrombocytosis and positive JAK2 mutation confirm the diagnosis of RARS-T.

\section{Conclusion}

RARS-T is an important and rare entity and have overlapping features of both refractory anemia with ringed sideroblasts and essential thrombocytosis making it difficult to diagnosis it accurately . It is largely a diagnosis of exclusion and bears a good prognosis. This case is rare and needs documentation to create awareness about overlap syndromes and prompt further research.

Funding: Nil, Conflict of interest: None initiated, Permission from IRB: Yes

\section{References}

1. Shimizu-Kohno K, Kimura Y, Kiyasu J, Miyoshi H, Yoshida M, Ichikawa R, Niino D, Ohshima $K$. Malignant lymphoma of the spleen in Japan: a clinicopathological analysis of 115 cases. Pathol Int. 2012 Sep;62(9):577-82. doi: 10.1111/j.1440-1827.2012. 02844.x.

2. Schnittger S, de Sauvage FJ, Le Paslier D, Fonatsch C. Refined chromosomal localization of the human thrombopoietin gene to $3 \mathrm{q} 27-\mathrm{q} 28$ and exclusion as the responsible gene for thrombocytosis in patients with rearrangements of 3q21 and 3q26. Leukemia. 1996 Dec; 10 (12):1891-6.

3. Thiele J, Kvasnicka HM. Clinicopathological criteria for differential diagnosis of thrombocythemias in various myeloproliferative disorders. Semin Thromb Hemost. 2006 Apr; 32(3) : 219- 230. PMID:16673276. DOI: $10.1055 / \mathrm{s}-2006-939433$

4. Tefferi A, Lasho TL, Schwager SM, et al. The JAK2 (V617F) tyrosine kinase mutation in myelofibrosid with myeloid metaplasia: lineage specificity and clinical correlates. Br J Haematol 2005; 131: 320 -328.DOI 10. 1111/i.1365-2141.2005.05776.x

5. Shaw GR, Ringed sideroblasts with thrombocytosis: an uncommon mixed myelodysplastic / myeloproliferative disease of elder adults, $\mathrm{Br} \mathrm{J}$ Haematol, 2005, 131 (2) : 180-184.

6. Steensma DP, Caudill JS, Pardanani A, McClure RF, Lasho TL, Tefferi A. MPL W515 and JAK2 V617 mutation analysis in patients with refractory anemia with ringed sideroblasts and an elevated platelet count. Haematologica. 2006 Dec;91(12 Suppl):ECR57.

7. Tatic A, Vasilică M, Coliţă A, Vasilache D, Dobrea C, Jardan C, Găman AM, Crişan AM, Coliţă D, Coriu D. Refractory anemia with ringed sideroblasts and thrombocytosis without JAK2 V617F mutation: report of three cases. Rom J Morphol Embryol. 2013; 54 (4) : 1177-82.

\section{How to cite this article?}

Omhare A, Singh SK, Srivastava K, Mishra V, Vahikar SU. A case report on MDS - MPD overlap syndrome - a diagnostic dilemma. Trop J Path Micro 2017;3(1):67-70.doi: 10.17511/jopm.2017.i1.12. 